\title{
II Congreso de Arqueología Peninsular
}

\author{
María R. Delgado Fernández ${ }^{1}$
}

Tras el éxito del I Congreso de Arqueologia Peninsular, celebrado en Oporto entre los dias 13 al 18 de octubre de 1993, Zamora ha tomado el relevo de esta ciudad para acoger el segundo encuentro entre los investigadores españoles y portugueses.

Dicho encuentro ha acontecido entre los días 24 y 28 de septiembre de 1996 en la capital zamorana con un rotundo éxito de participación, ya que el número de inscritos ha superado la cifra de quinientos.

Así pues, y continuando la línea ya trazada en el I Congreso, ha estado coordinado por dos investigadores españoles, los Profesores Rodrigo de Balbín y Primitiva Bueno de la Universidad de Alcalá de Henares, junto con Vitor Oliveira Jorge de la Universidad de Oporto, y ha contado con la ayuda y patrocinio de numerosas Instituciones y Entidades, especialmente la hispano-portuguesa Rei Afonso Henriques.

El Congreso se ha articulado en varias secciones, en concreto seis, que recogen comunicaciones referentes a Paleolítico-Epipaleolítico, Neolítico-Calcolítico-Bronce, Primer Milenio, Arqueología Clásica, Arqueología Medieval y Metodología.

Ante el amplio número de participaciones, todas las aportaciones no se han podido presentar en el marco del programa previsto en el Congreso, sobre todo cuando uno de los objetivos principales marcados por la organización ha sido fomentar el debate y la reflexión entre todos los investigadores peninsulares presentes. A nuestro parecer, en los criterios de selección han primado, sobre todo, el interés por cubrir la mayor parte de los ámbitos peninsulares presentados en cada sección y algo 
menos el tema o línea fundamental de la convocatoria del Congreso, allá por el mes de noviembre del pasado año, como era los trabajos que versarán sobre transiciones culturales o cuestiones generales que incitarán o propiciarán el debate.

Por otra parte, consideramos todo un acierto poder contar con la publicación de un Libro-Guía con los resúmenes de todas y cada una de las comunicaciones que se han presentado en este evento, de forma que se difunda sucintamente los trabajos aceptados por el comité asesor para la publicación en la Actas definitivas y así enriquecer el debate, aunque parece que éste último ha brillado más bien por su ausencia.

La Sección I (Paleolítico-Epipaleolítico) se desarrolló a lo largo del primer día y la mañana del 25, presentándose, aproximadamente, el $75 \%$ de las comunicaciones aceptadas y todas las contribuciones portuguesas. Hay que destacar, en primer lugar, la revisión cronoestratigráfica de la cueva de La Ermita (Hortigüela, Burgos) por parte de A. Moure, G. Delibes, I. Castanedo y Otros, cavidad excavada a principios de los ' 70 pero que, a partir de 1994, se han empezado a abordar los análisis polínicos, sedimentológicos, geocronológicos, industriales, etc. de una forma pormenorizada y sistemática.

Otras comunicaciones versaron sobre la transición entre Paleolítico Superior-Neolítico, como las aportaciones de P. Utrilla y C. Mazo para el Alto Aragón, E. Ramil Rego y J. Ramil Soneira para las Sierras septentrionales de Galicia o R. Mora, M. Pallarés y A. Bordás sobre el Noreste peninsular.

Igualmente, se han presentado datos recientes de los principales Proyectos de Excavación en curso, como Abric Romani (Capellades, Barcelona) por parte de M. Vaquero, N. Morant, X. Plana y Otros, ofreciendo los resultados de las últimas campañas. L. Raposo y J.L. Cardoso presentaban el contexto de Musteriense Final en Gruta Nova de Columbeira (Bombarral, Portugal) que coincide con otros contextos andaluces y levantinos, entrados en el Würm III, en claro contraste con las altas cronologías para el Paleolítico Superior Inicial que se tienen para el norte de la Peninsula.

Con respecto a la cueva de la Carihuela (Piñar, Granada), G. Vega y su equipo han presentado las industrias de la base de la secuencia conocida hasta ahora, siendo de gran interés por su carácter inédito. V. Cabrera, F. Bernaldo de Quirós y Otros se centraron en los niveles correspondientes al Auriñaciense Arcaico de la cueva del Castillo (Puente Viesgo, Cantabria), mostrando especial interés por la tecnología, viendo ciertos rasgos técnicos relacionables con el sustrato musteriense. 
También en esta línea, incluimos los trabajos de $P$. Arias y Otros en el complejo paleolítico de La Garma (Omoño, Cantabria), la presentación de los últimos trabajos en Anton Koba (Guipúzcoa) por parte de A. Armendáriz o los comentarios de $M$. Santonja de las últimas excavaciones en Torralba y Ambrona (Soria).

Por otro lado, se han revelado nuevos conjuntos de Arte paleolítico, como los recientemente descubiertos en la zona de Ramales por parte de C. González y C. San Miguel, algunas novedades puntuales de la cueva de El Buxu (Asturias) ofrecidas por M. Menéndez y P. Ocio, el arte de la cueva del Higueral (Cádiz) por parte de F. Giles y su equipo, provocando un intenso debate sobre la realidad de las figuras. Posiblemente, los trabajos presentados por J. Lejeune sobre la cueva de Escoural (Portugal) han sido uno de los más interesantes porque es la única cueva con decoración que se conoce hasta la actualidad en Portugal y para proponer una cronología relativa se ha tenido que recurrir a comparaciones con otras estaciones rupestres en cueva y al aire libre peninsulares y francesas.

Finalmente, otros temas seleccionados se han centrado en la definición o revisión del Paleolítico Superior en amplios sectores geográficos, como son los casos del Cantábrico y cuenca del Duero, por parte de F. Bernaldo de Quirós, A. Neira y C. Fernández y el caso más controvertido de la Meseta, comentado por J. Fabián, o temas más puntuales relacionados con análisis funcionales en materiales líticos como la comunicación de J.E. González y J.J. Ibáñez y las reconstrucciones experimentales de puntas microlíticas por parte de T. Aubri y Otros.

La Sección II (Neolítico-Bronce), celebrada a lo largo de las jornadas del 24 y 25 , ha girado en torno a cuatro grandes cuestiones: el hábitat, arte rupestre, mundo funerario y producción-distribución. Ha sido la sección más densa, con al menos 73 contribuciones, por lo que en el programa previsto ni siquiera han tenido cabida el $50 \%$ de las mismas, aunque la presencia portuguesa sí ha quedado bien reflejada.

En el ámbito del hábitat se incluyó en programa la presentación de asentamientos puntuales como $O$ Castro de Palheiros-Murça por parte de M.J. Sanches, el hábitat neolítico de Carriceiras por J. Senna-Martínez, el poblado de Amoreira por L. Oosterbeek y A.R. Cruz y el poblado de Monte da Ponte que presentaron $\mathrm{M}$. Hock y Ph. Kalb, haciendo hincapié en muchas de ellas en la conexión con el mundo megalítico, en contraposición a una aproximación al poblamiento diacrónico de un amplio territorio como es el Macizo de Cabra (Córdoba) por parte de B. Gavilán y su equipo.

En el ámbito de Arte Rupestre se admitió el trabajo de M.M. Ayala que daba a conocer unos nuevos planteamientos del Neolítico del Sureste ante 
los hallazgos de diversas cerámicas con unas decoraciones plásticas aplicadas que han sido interpretadas como representaciones de diosas y paralelizadas con la de Gavá (Barcelona).

En esta subsección dedicada al Arte Rupestre destacó especialmente la polémica surgida ante la posibilidad de valorar la autenticidad o no de algunos grabados de las cuevas de las Salinas (Soria) en concreto, presentada por J.A. Gómez Barrera, y de muchos conjuntos grabados a título general. De gran interés fue, también, la comunicación de $R$. Lucas y otros sobre el Arte Rupestre en el Barranco del Duratón (Segovia) y su posible atribución neolítica, los nuevos conjuntos esquemáticos documentados en Cáceres por A. González y M. de Alvarado y las nuevas manifestaciones de Arte esquemático en Cantabria por parte de L.C. Teira y R. Ontañón.

Uno de los capítulos mejor representados ha sido el funerario, siendo de especial relevancia las aportaciones de los investigadores portugueses al mundo megalítico en todos los ámbitos geográficos, en cambio, parece que el mundo funerario neolítico de filiación no megalítica sigue siendo poco conocido, pues únicamente se han presentado algunas puntualizaciones al controvertido grupo catalán de los "Sepulcros de Fosa" por parte de M. Martí y R. Pou.

Posiblemente, uno de los conjuntos megalíticos más destacables es el de los menhires de Pavía, presentado por L. Rocha, ya que se han detectado vestigios de hábitat en la zona que con el avance de las investigaciones en curso pueden llegar a aportar muchos datos novedosos con respecto a las relaciones hábitat-necrópolis. Por último, destacar algunas aportaciones de síntesis en el País Valenciano, como la de J.A. Soler, las novedades en Mallorca por V. Guerrero, J. Coll y M. Calvo o los enterramientos de carácter colectivo datados en contextos ya campaniformes que vienen apareciendo en Andalucia y en este caso concreto, en Málaga, como nos comentó J. Fernández.

Por último, en cuestiones de Producción y Distribución, hemos de destacar algunas propuestas de poblamiento en relación con determinados recursos mineros para la Edad del Cobre en Avila por parte de J. Fernández, J.J. Herrán, A. Orejas y Otros y en el País Valenciano a partir del II Milenio por J.L. Simón.

Ya inmersos en la Sección III, centrada en el Primer Milenio, ésta abarcaba la zona interior, mundo ibérico, área occidental y, finalmente, orientalizante, desarrollándose también entre los dias 24 y 25 en sesiones de mañana y tarde puesto que ha sido una de las más densas, con más de 60 comunicaciones aceptadas y algo más de la mitad de ellas incluidas en programa. 
El bloque seleccionado para al ámbito geográfico interior, se ha caracterizado, sobre todo, por las aportaciones puntuales a estudios ya conocidos, como son los trabajos geofísicos en el entorno de Numancia, a cargo de A. Jimeno, las dataciones radiocarbónicas de Cuéllar, presentadas por $J$. Barrio y realizadas por $F$. Alonso y estudios de materiales cerámicos, por parte de C. Sanz, o metálicos como el de P.J. Cruz y J. Quintana, salvo el trabajo desde la Arqueología del Paisaje que encabezaba G. Ruiz Zapatero y que, finalmente, no fue presentado.

El ámbito ibérico tampoco ha supuesto grandes novedades sino que más bien la tendencia ha sido presentar la puesta al día de trabajos en curso u otras puntualizaciones a estudios conocidos. Así pues, se han dado a conocer algunas novedades que se han producido en el Proyecto de $\mathrm{T}$. Chapa y J. Pereira en la Alta Andalucía, puntualizaciones a la escultura ibérica por parte de F. Quesada con respecto al conjunto de Porcuna y algunas manifestaciones inéditas de la provincia de Albacete aportadas por $\mathrm{J}$. Blánquez.

Siguiendo también una línea revisionista podemos incluir la comunicación referente a la necrópolis del LLano de la Consolación (Albacete), presentada por $\mathrm{C}$. Valenciano y algunas cuestiones nuevamente planteadas con relación a la escritura prelatina en el suroeste peninsular por parte de V. Correia.

En cuanto al área occidental, L. Berrocal planteó el estado actual del conocimiento con respecto a la defínición de la "Baeturia céltica", etnogénesis controvertida pero que, tras muchos años de investigación, parece ir identificando. Otras aportaciones han sido puntualizaciones concretas con respecto a la dispersión de materiales tipo Cogotas I por la Península y los problemas de identíficación de la misma, por parte de $F$. Abarquero $o$ en relación de éstos con la órbita tartéssica en el área vetona, como presentaron S. Celestino y A. Martín, también se incluyeron algunos avances con respecto a la tecnología metalúrgica en el noroeste presentado por B. Comendador.

Más novedosas, sin duda, son las aportaciones portuguesas a la definición del Bronce Final y transición al Hierro I en varias áreas geográficas que vienen siendo abordadas mediante prospecciones intensivas y que empiezan a arrojar cierta luz sobre el poblamiento en estas cronologías, tan mal definido hasta el momento, siendo los casos de Silva y Soares unos de los más destacables, junto al trabajo de Bettencourt, centrado en las estructuras de enterramiento, su distribución espacial y las relaciones con localizaciones de hábitat en el norte de Portugal.

Las aportaciones presentadas en el Congreso correspondientes al mundo Orientalizante han versado, en líneas generales, en torno a la 
presentación de nuevos poblados fechados a partir del siglo VII a.C. en la desembocadura del Ebro, como es el caso de F. Gracia y Otros y en las tierras interiores del Sureste, por J. López y F. Sala. En cambio, O. Arteaga volvió a recrearnos con la evolución de la línea de costa andaluza a partir del Bronce Final, trabajos desarrollados desde hace algunos años en colaboración con el Instituto Arqueológico Alemán.

Otros temas elegidos por su interés fueron las nuevas aportaciones de M. Torres al estudio de la interacción entre fenicios e indígenas a partir de las evidencias funerarias o las posibles delimitaciones fronterizas y regionales que ha establecido M. Ponte a partir de la distribución de ciertas fíbulas del Bronce Final.

Por otra parte, la Sección IV correspondiente a la Arqueología Clásica o tal vez, deberíamos decir Mundo romano exclusivamente, se desarrolló a partir del día 25 y ha centrado su atención en dos focos principales, por un lado, la ciudad y comercio y, por otro lado, la romanización y las evidencias en el territorio, suponiendo el programa un $50 \%$ de las comunicaciones aceptadas.

En líneas generales, se han presentado pocas cuestiones generales, tanto de carácter diacrónico como sincrónico que abarquen toda la Península o novedades metodológicas, en contraposición con los datos locales como resultados de excavaciones puntuales de yacimientos, siendo los casos del campamento de Petavonium (Zamora), presentado por S. Carretero y Otros, la ciudad de Labitolosa (Huesca) por A. Magallón, Sisapo (Ciudad Real), por M. Zarzalejos y C. Fernández.

Por tanto, ha resultado especialmente destacable los novedosos datos que sobre la Corduba Patricia presentó A. Ventura Villanueva, sorprendiendo a los asistentes por los espectaculares avances que está aportando el Proyecto Urbano de Córdoba y, del mismo modo, las cuestiones de cambio, adaptación o continuidad en la arquitectura hispana como consecuencia de la romanización que ilustraron brillantemente $M$. Bendala y $L$. Roldán.

Por su carácter novedoso, destacamos también el trabajo de $P$. Atoche y J. Paz que empiezan a hilvanar las evidencias romanas en Canarias para establecer un marco de relaciones entre el Mediterráneo y el conjunto insular.

De gran interés han resultado las comunicaciones que han versado sobre las cuestiones de romanización en diferentes territorios peninsulares, en la región valenciana, el noroeste, el Duero y el Alto Támega. En concreto, quisiéramos destacar la nueva visión de la Conquista del 
Noroeste peninsular presentada por A. Orejas y F.J. Sánchez pues hábilmente han sabido conjugar las fuentes literarias y arqueológicas.

Finalmente, bajo la denominación de Tipología y Tecnología se han incluido los trabajos numismáticos, las producciones de vidrio y otras aportaciones sobre materiales cerámicos, algunos de los cuales han puesto de manifiesto una cuestión importante y muchas veces olvidada como es la necesaria integración de todas las fuentes, ya sean numismáticas, literarias, epigráficas $o$ arqueológicas, en un estudio que pretende ser de reconstrucción histórica, destacando especialmente en este sentido la aportación de J.I. Cano Montero sobre evidencias arqueológicas de vidrio blanco y la contrastación con las fuentes escritas.

En dos sesiones de mañana y tarde, y a partir del día 24 , se desarrolló el programa correspondiente a la Arqueología Medieval, que agrupaba el mundo visigodo, feudal e islámico. Se incluyeron en programa el $66 \%$ de las comunicaciones aceptadas, siendo aportaciones portuguesas el $12 \%$ del total, de las cuales se incluyeron la mitad en el programa definitivo.

En líneas generales, no aventuraremos muchos comentarios sobre esta sección puesto que el medievalismo es ajeno y muy lejano a nuestras líneas de investigación.

Los temas presentados responden al panorama actual de la investigación medieval. Por un lado, situariamos el estudio del poblamiento o la estructuración de espacios agrarios, como es el caso de M. Fernández Mier aportando diversos ejemplos de Asturies o en cuanto a distribuciones espaciales de alquerias en Mallorca, por parte de M. Argemí e I. Navarro y Otros sobre el entorno de Bobastro, la organización territorial del distrito de Vélez-Málaga, por parte de $\mathrm{C}$. Delaigue o trabajos que se centran en la Marca Media Central-Occidental, como la contrastación de ámbitos rurales y urbanos andalusies o sus relaciones con la presencia de vias de comunicación, analizadas por L. Serrano, S. Martínez y P. Grañeda en un caso y A. Malalana, F. Saez, y S. Martínez en el segundo caso.

Por otro lado, agrupamos el estudio de materiales, especialmente los cerámicos, análisis de estructuras, como el alfar islámico de Elche, presentado por R. Azuar y J.L. Menéndez, decoración arquitectónica, junto a la hidráulica y su influencia en la elección de emplazamientos, como el trabajo de $\mathrm{C}$. Batet sobre algunos monasterios cistercienses. La Numismática completa el panorama, siendo destacables los trabajos preliminares de $A$. Canto y su equipo sobre monedas califales, lingotes de plata y otros fragmentos de moneda califal procedentes, todos ellos, de Haza del Carmen. 
Resultan de gran interés la presentación de algunas secuencias estratigráficas puntuales, como el yacimiento del cerro de la Virgen del Castillo (Segovia), por parte de J. Barrio o la lectura estratigráfica de Sta. María la Mayor (Valladolid), por C. Escribano.

En suma, supone una puesta al día de los trabajos en curso, ofreciéndose en algunos casos estudios de fondo sobre temas novedosos y en otros, puntualizaciones sobre cuestiones ya conocidas, siendo el caso de los nuevos trabajos urbanos en el entorno de la Medina de Málaga llevados a cabo por J. Suárez y Otros o los trabajos centrados en la pequeña fortaleza medieval en el yacimiento romano de Carteia que presentó S. Martínez.

Para finalizar, la Sección VI incluía nuevas metodologías y técnicas aplicadas a la arqueología. Como suele ser normal, esta sección tuvo una acogida sensiblemente menor, por lo que favoreció que se pudieran incluir en programa de sesiones el $80 \%$ de las comunicaciones. Esto también pudo favorecer el clima de debate y reflexión que surgió a propósito de las ventajas y los límites que tienen estas nuevas técnicas. En resumen, ¿qué se puede pedir a las nuevas metodologias?

En esta línea, hemos de destacar las propuestas de metodología arqueobotánica en yacimientos arqueológicos y los posibles resultados que se pueden obtener, tanto la propuesta por L. Peña y L. Zapata como la desarrollada por R. Buxó y N. Rovira.

Realmente impactante nos resultó el Proyecto Holmes (J.D. Sacristán y Otros) que desarrolla una metodología de estudio de impresiones dermopapilares sobre la arcilla de alfarero para poder determinar caracterizaciones bioantropológicas individuales.

Otros trabajos se centraban en análisis arqueometalúrgicos, como el de F.J. Sarabia, en el campo de las dataciones absolutas, la Traceologia, la interpretación funcional de los instrumentos artefactos, especialmente el estudio de fitolitos presentado por $\mathrm{J}$. Juan o la caracterización de cerámicas.

Por otro lado, aquellos investigadores que quisieran presentar de una forma gráfica los trabajos en curso de un yacimiento, los objetivos o fases que se están desarrollando en un Proyecto o cualquier otro aspecto de interés han tenido la posibilidad de exponer esta información en forma de Poster, incluyéndose en programa un horario de presentación y comentarios de los mismos ante cualquier consulta de los congresistas, decisión que es realmente de agradecer, aunque parece que este tipo de iniciativas aún no cuenta con una buena acogida entre nuestro colectivo.

Dado el apretado programa previsto, consideramos todo un acierto la inclusión de una jornada vespertina de excursiones durante el desarrollo 
del Congreso por los alrededores cercanos de Zamora para visitar las principales joyas del Arte visigodo y del románico zamorano. También se ha contemplado una excursión Post-Congreso a las estaciones de Arte al aire libre de Siega Verde y Foz-Côa, dos de los conjuntos de grabados paleolíticos más significativos del interior de la Península, junto al indiscutible de Domingo Garcia (Segovia).

Únicamente quisiéramos resaltar como imponderables lógicos en una reunión de estas características los cambios y las alteraciones de programa ante la ausencia de comunicantes que habían confirmado su presencia. Esto es una realidad que, desgraciadamente, cada vez viene siendo más frecuente en los Congresos con los inevitables desajustes y descalabros para la organización y para los congresistas que ven realmente complicada la tarea de asistir o compaginar el desarrollo de varias secciones a la vez.

Por otro lado, consideramos necesario llevar a cabo una selección de las comunicaciones que se van a presentar en las diferentes secciones por razones obvias ya comentadas pero creemos que esta selección sería más rigurosa si se hiciera sobre la comunicación íntegra y no sobre resúmenes de cinco líneas, que apenas dejan vislumbrar el enfoque de la aportación. De esta forma, el Comité de selección sería más operativo y se podrian solventar en gran medida los problemas de recepción de los textos para la ulterior publicación.

Ya para terminar, sólo nos queda felicitar a la Organización por la decisión de continuar con la iniciativa de encuentros peninsulares, la comunicación fluida que ha existido entre la misma y los investigadores interesados durante la gestación y preparación del encuentro y la nada parca información recibida a lo largo de los meses que han pasado entre la convocatoria y la celebración del mismo. 